\title{
Social Media as a Tool for Misinformation and Disinformation Management
}

\author{
Ibegbulem Obioma Hilary \\ Department of Business Administration and Marketing, Faculty of Social Science, \\ Delta State University, Abraka, Nigeria \\ Olannye-Okonofua Dumebi \\ Department of Mass Communication, Faculty of Social Science, Delta State \\ University, Abraka, Nigeria
}

\begin{abstract}
In recent years, the subject of fake news, as well as its consequences, has gained a lot of attention. Even though fake news is not a new occurrence, technological advancements have created an ideal atmosphere for it to spread quickly. Platforms like Facebook, Twitter, and YouTube provide fertile ground for the creation and dissemination of misinformation and disinformation. As a result, it is critical to research how social media works, how fake news is created and distributed through social media, and what role users play. The study examines social media as a tool for misinformation and disinformation. Been qualitative, the paper relies on secondary data such as published materials and personal observations to make deductions and inferences about the use of social media for fake news. This study examines misinformation and disinformation as a kind of fake news, as well as the many sorts of misinformation that may be found on social media. It adds to the idea of fake news by addressing the problem of users' interactions with news and cooperation in the information age. To add credibility to the study, the idea of misinformation and disinformation was investigated. In addition, the role of social media in the spread of misinformation and disinformation was studied to provide a thorough framework for the study. The study concluded with recommendations for preventing information manipulation on social media.
\end{abstract}

Keywords---disinformation, fake news, misinformation, social media.

Linguistics and Culture Review (c) 2021.

Corresponding author: Hilary, I. O.; Email: hilaryibegbulem27@gmail.com

Manuscript submitted: 09 April 2021, Manuscript revised: 27 July 2021, Accepted for publication: 18 August 2021 496 


\section{Introduction}

The sort of news people get has changed dramatically because of the Internet. Individuals formerly relied on conventional media such as radio and television, which included fewer and more well-established news sources. Individuals are increasingly exposed to online sources of information, such as social networking sites, which allow anybody to publish anything without the need for "factchecking or editorial judgment" (Allcott \& Gentzkow, 2017). Many people are concerned that internet sites may post misleading material while presenting it as "genuine" news. More individuals are getting involved with social media as a result of the growing popularity of a wide range of internet-enabled gadgets and enhanced mobile internet speeds. Indeed, Facebook is used by most people globally, and many of us obtain our news from social media posts (Stephen, 2016; Erdoğmuş \& Cicek, 2012).

Given the recent focus on the role of social media in the spread of fake news regarding current political and social events, it is critical to comprehend how the public interacts with misinformation on social media platforms (SNS). Fake news concerning contemporary social and political concerns spreads at a breakneck speed on social media (Koohikamali \& Sidorova, 2017). These hoaxes or bogus tales misinform or deceive audiences, whether on purpose or unintentionally. These tales are usually made to either influence people's opinions, push a political agenda, or confuse people, and they may be lucrative business for internet publishers (Brennen, 2017). Platforms like Facebook and Twitter have brought us closer together in new and powerful ways. Stories and views may spread at an unparalleled rate, allowing people all over the world to participate in a near-real-time discussion on both serious and frivolous issues.

Take, for example, Micheal, who is concerned about catching COVID-19. He relies on trustworthy people for advice because he is unable to read all the items he sees on it. When someone suggests on Facebook that pandemic worries are exaggerated, Micheal first dismisses the notion. When the hotel where Micheal works closes its doors, and with his job on the line, he begins to doubt how severe the new virus's danger is. After all, no one he knows has died. Micheal's skepticism of government is echoed by a colleague's tweet about the COVID "scare" being generated by Big Pharma in conjunction with unscrupulous politicians. His Internet search yields publications suggesting that COVID-19 is no more dangerous than the flu. Micheal joins an online community of people who have been laid off or are about to be laid off, and like many of them, he quickly finds himself thinking, "What pandemic?" He chooses to attend a demonstration demanding an end to lockdowns after learning that some of his new pals are going to attend. Almost no one, including him, is wearing a mask at the big demonstration. When his sister inquires about the protest, Micheal expresses his belief that COVID is a deception, which has now become a part of his identity.

This illustration shows a minefield of cognitive biases. We prefer to get information from individuals we know and trust, members of our in-group. We pay attention to and are more inclined to share information concerning dangers, such as Micheal's job loss. However, modern technologies exacerbate these biases 
in negative ways. Micheal's concerns are stoked by search engines, and social media connects him with others who share his anxieties. Worse, bots-automated social media accounts that imitate people-allow confused or malicious actors to exploit their flaws (Altam, 2020; Tomas \& Dulin, 2021). The abundance of internet information only exacerbates the situation. The cost and ease of viewing and generating blogs, movies, tweets, and other information units known as memes has resulted in an overabundance of information on the market (Menczer \& Hills, 2020).

\section{Vulnerability to Fake News}

A study of Twitter users that rated their political leanings found that both liberals and conservatives end up sharing information from sites that repeatedly post news of low credibility (as identified by independent fact-checkers). Conservative users are somewhat more susceptible to sharing fake news, however.

More than 15,000 Twitter users are plotted on this matrix. The size of each dot represents the number of accounts that share that political bias/misinformation coordinate, ranging from one to 429 .

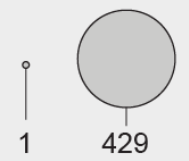

Political Bias of Twitter Users (inferred by set of news sources shared by user)

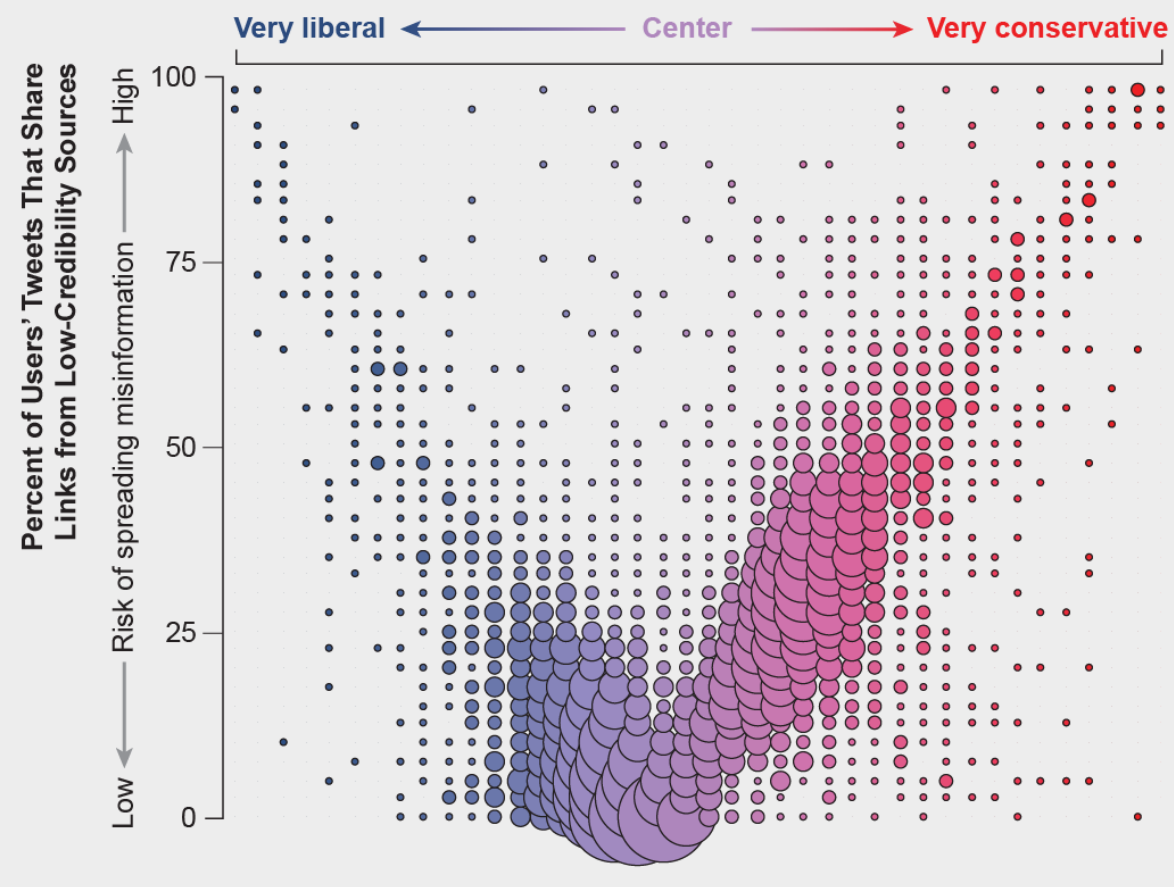

Figure 1. Vulnerability to fake news Source: Dimitar Nikolov and Filippo Menczer (data) 
A similar dynamic exists in social media. We conflate popularity with excellence, and we end up imitating what we see. Bjarke Mnsted and his colleagues at the Technical University of Denmark and the University of Southern California found that knowledge is spread by "complex contagion": when we are frequently exposed to an idea, generally from a variety of sources, we are more likely to accept and reshare it. This social bias is exacerbated by the "mere exposure" effect, which occurs when people are repeatedly exposed to the same stimuli, such as specific faces, and develops to prefer those stimuli over others they have seen less frequently.

Such biases result in an insatiable need to pay attention to material that has gone viral-if everyone else is talking about it, it must be significant. Social media sites such as Facebook, Twitter, YouTube, and Instagram, in addition to displaying stuff that aligns with our beliefs, display popular material at the top of our screens and show us how many people have liked and shared something. Few people are aware that these cues do not give impartial quality judgments. (Menczer \& Hills, 2020). The goal of this research is to examine how social media serves as a tool for misinformation and disinformation (Sessions et al., 2003; Colliander, 2019).

\section{Misinformation vs disinformation}

When considering what constitutes "fake news" and how it is spread on social media, there are two sorts of false information to be aware of. According to Indiana University researchers, these two types of information commonly go viral because "information overload and users' short attention span impair social media's ability to discriminate material based on quality." Because social media is a public forum, anybody may post whatever they want, including news organizations, without fear of being held accountable for fact-checking (Pennycook \& Rand, 2019; Bondielli \& Marcelloni, 2019). Users must decide if their feeds include false or misleading information.

"Misinformation" and "disinformation" are not synonyms, even though they are frequently used interchangeably. Inaccurate reporting resulting from errors is referred to as misinformation; nevertheless, the phrase does not indicate deception. On the other hand, misinformation. Refers to the deliberate dissemination of false information to deceive others. Disinformation is always misinformation, but misinformation can sometimes be disinformation, depending on the aim (Ecker et al., 2017; Erku et al., 2021). It is disinformation, for example, when a factual inaccuracy is discovered in an article about a political figure (Nikolov, 2020). The article might be labeled misinformation if it is discovered that the error was deliberate. Because determining intent can be difficult, these words are frequently used interchangeably.

\section{Misinformation}

Misinformation refers to those who spread false information without realizing it, usually because their friends or others do (Campan et al., 2017). The echo chamber effect greatly contributes to this aspect; the social media system is made 
up of an algorithm that recommends certain news or information to a consumer based on the group to which he or she belongs on social media, their prior history, and circle of friends, such that when one friend views something, another friend is recommended the same thing, and the user is notified of such corecommendation. This recommendation system also works as a motivator for consumers to share information, even if they are unsure of its authenticity. People who share the same beliefs or belong to the same political party will distribute and share information that is favorable to their political goals without verifying it.

Humans are typically bad at recognizing what is genuine and what is legitimate, according to cognitive theories (Vaccari \& Chadwick, 2019), and they are prone to fake news because of their credulous nature. Vaccari \& Chadwick (2019), argue that individuals are more likely to accept anything that confirms their beliefs (confirmation bias) and would spread it without verifying it because it aligns with their thinking while distorting those that do not, even if they are true. The aim of the person or source disseminating the information distinguishes misinformation from disinformation. It is not the intention of misinformation to deceive (Aldwairi \& Alwahedi, 2018; Di Domenico et al., 2021). Instead, it seeks to influence or modify public opinion on a particular issue.

\section{Types of misinformation on social media Clickbait}

Clickbait is sensationalized material or headlines that are meant to pique readers' interest by appealing to their emotions (usually anger) or curiosity. The goal of clickbait, as the term implies, is to get readers to engage with the material to earn ad income. It is generally devoid of facts or other useful information, instead of spreading them thin material across several pages to maximize the number of adverts that may be delivered to each user. If that was not bad enough, clickbait may also disseminate false information. Readers may become enraged and share this badly researched and written content with their social connections, spreading incorrect information to an increasing number of people (Lipschultz, 2020).

\section{Misleading titles}

Misleading titles, like clickbait, may be used to pique our interest by appealing to our emotions and curiosity. Even if the piece is well-written and factually accurate, the content might convey the wrong impression if the headline is deceptive. As a result, even if they don't match the criteria of "clickbait" stated above, such headlines may be referred to as "clickbait." Unfortunately, according to studies, most social media users share stories based on the title without really reading the text. If the title does not correctly reflect the reality, it may have the same impact as false articles (Lipschultz, 2020).

\section{Propaganda}

Propaganda is biased and potentially misleading information that is disseminated through the media with the intent of persuading its target audience to adopt certain viewpoints. Propaganda employs a variety of strategies, including: 
- Emotional appeals: Propaganda, like other types of disinformation, frequently relies on emotional appeals to pique readers' attention and influence them.

- Using lies or Partial truth: Propaganda may use blatant lies or "half-truths" to persuade readers. To support a certain point of view, it may employ incomplete quotations, distorted data, or other dishonest tactics.

- Testimonials: To promote their point of view, propaganda publications may include testimonials, often from well-known celebrities. These are occasionally manufactured as well.

Political material frequently employs emotive appeals, presents distorted information, and relies on endorsements from well-known celebrities and officials (Lipschultz, 2020).

\section{Satire}

News satire is a form of parody that imitates genuine news sources and derives its comedy from its deadpan, sarcastic delivery. Because not every reader will see the irony, satire like this might be mistaken as reality. While satire is not meant to be manipulative, it may have the same impact as false news if it's not read correctly (Lipschultz, 2020).

\section{What role do social media users play in spreading misinformation?}

Misinformation has thrived on social media during the last decade. While information about users, such as demographics and internet usage, is meant for advertisers, it may also be utilized by those seeking to disseminate disinformation. Fake news can be crafted to appeal to a core audience using this information to generate genuine participation. From there, the content can quickly gather traction, with the possibility of turning viral. As a result, social media expands the reach of individuals spreading false information. Each social media platform adds to disinformation in its unique way, influencing how people approach subjects such as politics, health, and other topics.

\section{Disinformation}

Many of the same methods that are used to propagate misinformation may also be used to spread disinformation: hoaxes, clickbait, and falsified stories. The purpose of disinformation is to deceive. According to Chadwick \& Vaccari (2020), 24.8 percent of their respondents shared a news item that they either felt was made up or recognized was overblown when they first saw it. Individuals' social media accounts, as well as company accounts, may propagate misinformation for a variety of reasons. It might be to improve their social media marketing efficacy, increase internet traffic, increase page or business followers, elicit an emotional response, or create a diversion. Disinformation is harmful on social media because, as previously said, the vast volume of information available and the short attention spans of readers can allow it to spread unchecked. 


\section{The power of social media}

Understanding the intentions of fellow posters and the site itself is key to combating fake news on social media. Users' data is sold to ad firms by social media platforms, which is why you will often see advertising targeted to your interests or search history. This is crucial to understand considering the situation. Knowing that the news you receive on your feed is filtered based on previously collected data might help you become more aware of your own inherent bias as a person. If you work for a company that uses social media as a marketing tool, it is critical to maintain your postings consistent with your brand and publish items on your timeline that help you create customer connections, align with your beliefs, or highlight unique material.

It may be impossible to avoid fake news on social media. However, critical thinking might assist to halt the spread. Maintain a healthy degree of interest in what you see on your feed, learn how social media sites control what you see, and employ investigative techniques often. When addressed with the right aim and thought, social media can be a tremendous tool for both organizations and individuals.

\section{Curbing social media information manipulation}

We can better protect ourselves against manipulation by understanding our cognitive biases and how computers and bots exploit them. Some have developed a variety of tools to assist users in recognizing their vulnerabilities as well as the flaws in social media networks. One is Fakey, a smartphone app that teaches users how to recognize false information. The game is a simulation of a social media news feed, including real items from low- and high-credibility sources. Users must determine what they may and cannot share, as well as what they should fact-check.

Another publicly available software, Hoaxy, demonstrates how any existing meme spreads on Twitter. Nodes reflect genuine Twitter accounts, and linkages show how the meme spreads from account to account through retweets, quotations, mentions, and responses. Users may observe the scale at which bots multiply disinformation by looking at the color of each node, which represents its Botometer score. Investigative journalists have utilized these methods to discover the origins of disinformation campaigns, such as the one promoting the "pizza gate" theory in the United States. During the 2018 U.S. midterm elections, they also assisted in the detection of bot-driven voting suppression operations (Menczer \& Hills, 2020).

However, as machine-learning algorithms improve in simulating human behavior, manipulation is becoming more difficult to detect. Misinformation campaigns can serve as a distraction from other, more significant issues in addition to disseminating false information. We just released BotSlayer, a software tool designed to counteract such exploitation. It pulls hashtags, URLs, accounts, and other elements that appear often in tweets on topics that the user wants to learn more about. BotSlayer analyzes tweets, accounts that publish them, and bot 
scores for each entity to identify entities that are trending and are likely being amplified by bots or coordinated accounts.

These programmatic tools are helpful, but institutional reforms are also required to prevent the spread of fake news. Although education can assist, it is unlikely to cover all the subjects where individuals are misinformed. Online manipulation and false news are also being targeted by certain governments and social media sites. But who judges what is and isn't false or manipulative? Warning labels, such as those provided by Facebook and Twitter, can accompany information, but can the people who apply those labels be trusted? The danger that such restrictions would unintentionally or intentionally stifle free expression, which is essential for strong democracies, is significant.

The dominance of global social media platforms with tight links to governments further complicates the options. Making it harder to generate and spread lowquality material could be one of the finest ideas. This might include creating friction by requiring individuals to pay to share or receive data. Time, a mental effort such as riddles, or tiny costs for subscriptions or usage might all be used as payment. Automated posting must be regarded as if it were a kind of advertising. To get access to accounts, several sites already employ friction in the form of CAPTCHAs and phone verification (Menczer \& Hills, 2020).

Automated posting on Twitter has been restricted. These initiatives might be broadened to progressively shift internet sharing incentives toward consumervalued content. Free communication does not imply that it is unrestricted. We have reduced the value of information and fostered its adulteration by lowering its cost. We must understand the vulnerabilities of our overburdened brains and how the economics of information may be used to prevent us from being misled to restore the health of our information environment.

\section{Conclusion}

The paper, qualitative relied on secondary data such as published materials and personal observations to make deductions and inferences about the use of social media for fake news. The emphasis was on examining misinformation and disinformation as a kind of fake news, as well as the many sorts of misinformation that may be found on social media. The findings exposed the underlying dangers of misinformation with suggestions on how best to address this social communication menace.

\section{Recommendation}

- The regular use of technological tools in fast checking online information

- Government to continue to devise better policies to check the online circulation of false news. 


\section{References}

Aldwairi, M., \& Alwahedi, A. (2018). Detecting fake news in social media networks. Procedia Computer Science, 141, 215-222. https://doi.org/10.1016/j.procs.2018.10.171

Allcott, H., \& Gentzkow, M. (2017). Social Media and Fake News in the 2016 Election.(January 2017). NBER Working Paper, (23089).

Altam, S. . (2020). Influence of social media on EFL Yemeni learners in Indian Universities during Covid-19 Pandemic. Linguistics and Culture Review, 4(1), 35-47. https://doi.org/10.37028/lingcure.v4n1.19

Bondielli, A., \& Marcelloni, F. (2019). A survey on fake news and rumour detection techniques. Information Sciences, 497, 38-55. https://doi.org/10.1016/j.ins.2019.05.035

Brennen, B. (2017). Making sense of lies, deceptive propaganda, and fake news. Journal of Media Ethics, 32(3), 179-181.

Campan, A., Cuzzocrea, A., \& Truta, T. M. (2017, December). Fighting fake news spread in online social networks: Actual trends and future research directions. In 2017 IEEE International Conference on Big Data (Big Data) (pp. 4453-4457). IEEE.

Colliander, J. (2019). "This is fake news": Investigating the role of conformity to other users' views when commenting on and spreading disinformation in social media. Computers in Human Behavior, 97, 202-215. https://doi.org/10.1016/j.chb.2019.03.032

Di Domenico, G., Sit, J., Ishizaka, A., \& Nunan, D. (2021). Fake news, social media and marketing: A systematic review. Journal of Business Research, 124, 329-341. https://doi.org/10.1016/j.jbusres.2020.11.037

Ecker, U. K., Hogan, J. L., \& Lewandowsky, S. (2017). Reminders and repetition of misinformation: Helping or hindering its retraction?. Journal of Applied Research in Memory and Cognition, 6(2), 185-192. https://doi.org/10.1016/j.jarmac.2017.01.014

Erdoğmuş, İ. E., \& Cicek, M. (2012). The impact of social media marketing on brand loyalty. Procedia-Social and Behavioral Sciences, 58, 1353-1360. https://doi.org/10.1016/j.sbspro.2012.09.1119

Erku, D. A., Belachew, S. A., Abrha, S., Sinnollareddy, M., Thomas, J., Steadman, K. J., \& Tesfaye, W. H. (2021). When fear and misinformation go viral: Pharmacists' role in deterring medication misinformation during the'infodemic'surrounding COVID-19. Research in Social and Administrative Pharmacy, 17(1), 1954-1963. https://doi.org/10.1016/j.sapharm.2020.04.032

Koohikamali, M., \& Sidorova, A. (2017). Information Re-Sharing on Social Network Sites in the Age of Fake News. Informing Science, 20.

Lipschultz, J. H. (2020). Social media communication: Concepts, practices, data, law and ethics. Routledge.

Menczer, F., \& Hills, T. (2020). Information overload helps fake news spread, and Social Media Knows It. Scientific American, 323(6), 54-61.

Nikolov, D., Flammini, A., \& Menczer, F. (2020). Right and left, partisanship predicts (asymmetric) vulnerability to misinformation. arXiv preprint arXiv:2010.01462.

Pennycook, G., \& Rand, D. G. (2019). Lazy, not biased: Susceptibility to partisan fake news is better explained by lack of reasoning than by motivated 
reasoning. Cognition, 188,

39-50.

https:/ / doi.org/10.1016/j.cognition.2018.06.011

Sessions, A. E., Rabinowitz, R., Hulbert, W. C., Goldstein, M. M., \& Mevorach, R. A. (2003). Testicular torsion: direction, degree, duration and disinformation. The Journal of urology, 169(2), 663-665. https://doi.org/10.1016/S0022-5347(05)63987-0

Stephen, A. T. (2016). The role of digital and social media marketing in consumer behavior. Current opinión in Psychology, 10, 17-21. https://doi.org/10.1016/j.copsyc.2015.10.016

Tomas, R. R. S., \& Dulin, A. T. (2021). Social media exposure and paragraph writing of pre-service students. Linguistics and Culture Review, 5(S3), 144-153. https://doi.org/10.37028/lingcure.v5nS3.1379

Vaccari, C., \& Chadwick, A. (2020). Deepfakes and disinformation: Exploring the impact of synthetic political video on deception, uncertainty, and trust in news. Social Media+ Society, 6(1), 2056305120903408.

Vaccari, C., \& Chadwick, A. (2020). Deepfakes and disinformation: Exploring the impact of synthetic political video on deception, uncertainty, and trust in news. Social Media+ Society, 6(1), 2056305120903408. 